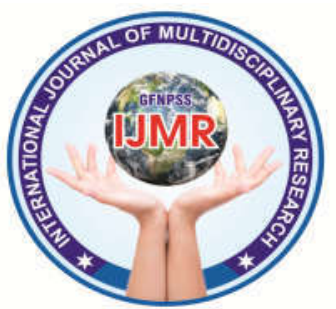

\title{
AN IN VIVO COMPARISON OF POST-OPERATIVE PAIN AFTER SINGLE VISIT VERSUS MULTIPLE VISIT ROOT CANAL THERAPY BY ROTARY AND HAND FILES
}

\author{
DR. Y.S. HADA \\ DEPARTMENT CONS AND ENDO., DASWANI DENTAL COLLEGE KOTA, \\ RAJASTHAN
}

Corresponding Email: coolhadays@rediffmail.com

\begin{abstract}
Background- ..comparison of post obturation pain after single visit and multiple visit endodontic treatment.
\end{abstract}

Material and Method:After ethical approval, proper consent form patients and diagnosis 100 teeth with irreversible pulpitis were selected and randomly divided in two groups, Group A(GA) and Group B (GB) consisted of 50 teeth each to be treated in a single visit and multiple visits respectively and which were further divided into two groups GA1(Hand Protapers) and GA2 (Rotary Protapers), GB1 (Hand Protapers) and GB2 (RotaryProtapers), respectively consisting $\mathrm{n}=25$ teeth each group. Teeth in Group GA a after proper debridement of canal with Hand or Rotary Protapers were obturated during the initial appointment but teeth in Group GB after proper debridement of canal with hand or Rotary Protapers were sealed without any intracanal medicament application with a sterile dry cotton pellet and temporary filling material. Patients were recalled and obturated after proper irrigation, in another appointment.. Pain was recorded on the visual analogue scale after postoperative period of $6 \mathrm{hrs}, 24 \mathrm{hrs}, 48 \mathrm{hrs}$, and 1 week. Each patient was given a prescription for $600 \mathrm{mg}$ of Ibuprofen tablets to be taken 8 hourly with instructions to avail the same only if needed for pain. Data was compiled and statistically analyzed.. Multiple comparisons of pain in different subgroups was done using Mann-Whitney Test and Kruskal-Wallis Test .

Result :Comparing the results for single visit root canal therapy (group GA) with multiple visit root canal therapy (group GB), statistically significant difference was found in post obturation pain. 


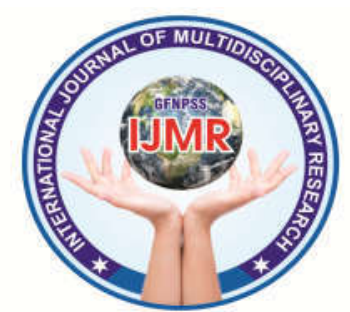

Conclusion: In Group GA, significantly more post obturation pain was there in group GA1 as compared to group GA2.In Group GB, no significant difference was found in post obturation pain in GB1 as compared to GB2.

Keywords : Hand Protaper, Rotary Prpotaper, Single visit RCT, Multiple visits RCT.

\section{INTRODUCTION:}

Endodontic treatment comprises of three main phases: biomechanical preparation of the root canal (cleaning and shaping), disinfection and obturation. ${ }^{1}$ Dr. Grossman advocated root canal treatment to be performed in multiple visits primarily to ensure sterility of root canal system prior to obturation. ${ }^{1,2}$ With the advent of rotary NiTi instruments, mechanical preparations are faster than stainless steel hand instruments ${ }^{3}$, the canal is more centered, tapered, and better maintained in their original anatomy. ${ }^{5,6}$ Thus, improved and better forms of biomechanical preparation are being developed which include the coronal to apical approach techniques having advantages like less debris extrusion and elimination of coronal interferences. ${ }^{7}$ Because of all the above improvements, single visit endodontics is no more an orthodox empirical procedure but is considered as an acceptable alternate procedure for endodontic problems. ${ }^{4}$
One-visit root canal treatment compared to multi-visit treatment allows a reduction in the number of patient appointments, prevention of root canal contamination and bacterial re-growth that can occur when the treatment is prolonged over an extended period, less chances of inter-appointment flare up due to leakage or loss of temporary seal, immediate esthetic replacement for anterior teeth, familiarity of canal anatomy, constant working length.

Long term success of endodontic treatment is dependent on case selection, treatment procedures and protocols, time management and duration of appointments. Although, post operative pain is not the criteria for success of endodontic treatment, some patients might consider postoperative pain and flare-up as a benchmark against which the clinician's skills are measured. ${ }^{9}$

Fear of post operative pain is the biggest factor in avoiding single visit endodontic therapy. A large number of 


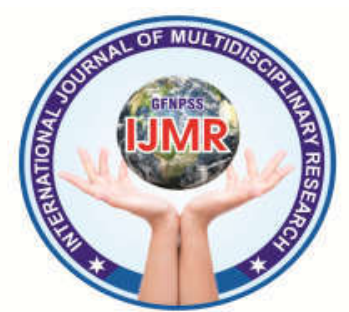

studies and research has been carried out to compare the incidence of post-operative pain in single visit versus multi-visit endodontic therapy. Hence, this study was undertaken to compare the incidence of post obturation pain during single visit root canal therapy versus multiple visit root canal therapy with hand and rotary files

\section{MATERIAL AND METHODS:}

SOURCE OF DATA: Patients who were coming for root canal treatment conducted in the Department of Conservative Dentistry and Endodontics, Daswani Dental College and Research Centre, Kota, Rajasthan.

Patient selection was done from who required Root Canal Therapy as treatment modality. From patients aged between 18 years to 60 years with the mean age of 35 39 years were selected for the study following the inclusion criteria tooth should have uncomplicated canal with fully formed apex, patients having periapical lesion having radiolucency less than $0.5 \mathrm{~cm}$, vital teeth and exclusion criteria medically compromised patients, TMJ problems and having problems with mouth openings, Under 18 years of age, Using antibiotics or corticosteroids, non restorable and periodontal compromised teeth, calcifications of teeth, internal and external resorption cases, mentally disabled patients, pregnant patients. Root canal therapy and the study were explained to the patients. Oral and written informed consent was obtained from all study participants. Detailed medical and dental history was obtained. Evaluation of pain experienced by the patients was done with a $100 \mathrm{~mm}$ Modified Heft - Parker visual analogue scale.

\section{METHODOLOGY GROUPING OF THE PATIENTS}

Total 100 teeth, which fulfilled the inclusion and exclusion criteria were selected, the patients were randomly assigned to either the one visit (Group A) or multiple -visit (Group B) before initiation of treatment: -On the basis of appointment given: Group A (GA) consisted of fifty teeth to be treated in a single visit which was further divided into two groups GA1 and GA2 consisting 25 teeth each to be treated by Hand Protaper and Rotary Protaper Files. Respectively. Group B (GB) consisted of fifty teeth to be treated in multiple visits which was further divided into two groups $\mathrm{GB} 1$ and $\mathrm{GB} 2$ consisting 25 teeth each to be treated by Hand Protaper and Rotary Protaper Files respectively. 


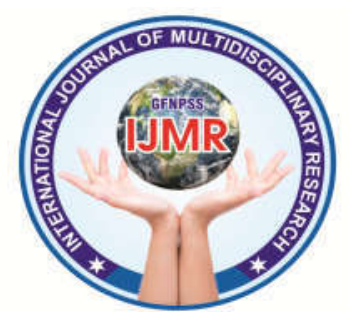

Once the proper consent obtained from the patient. The standard procedure for root canal treatment for both groups was done . Which was included pain control and isolation ( rubber dam,Hygienic, Coltene /Whaledent Inc. Germany) after this standard access cavity was prepared using the air roter and no .2 and no. 4 diamond burs. Endo Z Bur (Dentsply, Maillefer) was used to remove the overhanging dentin and enamel ,to obtain the straight line access to the root canal.

On the removal of the entire roof of the pulp chamber the coronal pulp can be visualized pulp extirpated and orifice localized. Then orifice openers were used for enlarging the coronal third of the canal. $15 \%$ EDTA used as a lubricant and 3\% $\mathrm{NaOCl}$ and saline were used as irrigants.

The working length of each canal was determined by an electronic apex locator or 2 or more angled radiographs. ${ }^{6}$

Biomechanical preparation of canal preparation was done as following way:(a) canals in group GA1 and GB1 were prepared by using hand protapers SX, $\mathrm{S} 1, \mathrm{~S} 2, \mathrm{~F} 1$ to $\mathrm{F} 5$.

(b) Canals in Group GA2 and GB2 were prepared using combination of Rotary protapers SX, S1, S2, F1 to F5 were used with Endo mate DT with gear reduction handpiece $20: 1$ at speed of $250 \mathrm{rpm}$. For SX torque 4, S1 torque 3, S2 torque 1.5, F1 torque 1.5, F2-F5 torque 2 was selected. All instruments were rotating when inserted in root canal. ${ }^{6}$

All instrumentation was done in wet canal with pulp chamber brimful with $3 \%$ $\mathrm{NaOCl}$. All instruments were liberally coated with Glyde. Irrigation was done with $2 \mathrm{ml}$ of $3 \% \mathrm{NaOCl}$ after each instrument.After completion of canal instrumentation, all canals were irrigated with $2.0 \mathrm{ml} \quad 17 \%$ ethylene diamine tetraacetic acid for 1 minute followed by final irrigation with $2.0 \mathrm{ml}$ of $3 \%$ sodium hypochlorite. After instrumentation and final irrigation, canals were dried with paper points. Obturation of teeth in Group A were obturated during the initial appointment with gutta percha cones and Zinc Oxide Eugenol sealer.

Teeth in Group B were sealed with a sterile dry cotton pellet and temporary filling material (e-TempTM, DiaDent Group International, Korea) . Patients in Group B were recalled for the further appointments one week later and the teeth were obturated with the same methods and materials used as in Group A.

All patients were prescribed mild painkillers (Ibuprofen), to be taken 8 


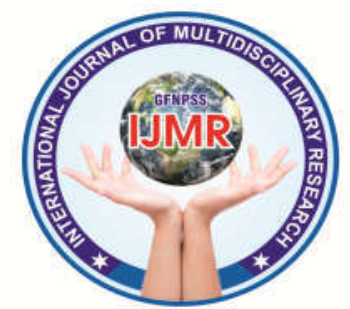

hourly only if they experienced moderate pain. Any of the patients with intolerable pain was instructed to visit the clinician for emergency treatment.

The clinical evaluation of post obturation pain was done with the $100 \mathrm{~mm}$ Modified Heft -Parkar Visual Analogue Scale (VAS).

The post-operative evaluation was recorded as:

0 - No pain

1 - Slight pain/discomfort

2 - Moderate pain relieved by analgesics

3 - Moderate to severe pain not completely relieved by analgesics

4 - Severe pain/swelling not relieved by analgesics and required unscheduled visit.

The patient carried the visual analogue scale form along with them. Pain was recorded by patient on the scale after postoperative period of $6 \mathrm{hrs}, 24 \mathrm{hrs}, 48 \mathrm{hrs}$, and 7 days. Reminder was given to them telephonically to note their pain readings. After 1 week of obturation, final clinical evaluation for pain was done with the vertical percussion method. At the same time all patients were asked to report any other reactions they felt till that day. Modified Visual analogue scales and record of medications taken were collected from the patients on this day. The data was compiled and statistically analyzed.

\section{RESULTS:}

Data was collected and results were statistically analyzed using KruskalWallis Test Differences were considered significant when the probabilities were equal to or less than 0.05 . The statistical analysis was done using SPSS (Statistical Package for Social Sciences) Version 17.0 statistical Analysis Software. Multiple comparisons of pain in different subgroups was done using Mann-Whitney Test.

Teeth in subgroups GA1 and GB1 were prepared using hand instruments and teeth in subgroups GA2 and GB2 were prepared using Endomotor and rotary instruments. These teeth were assessed for incidence and intensity of post obturation pain, swelling, time taken to complete the procedure. None of the patients reported swelling or need for antibiotics.

Table 1 : Statistical evaluation of pain scores in groups ( $G_{A}$ and $\left.G_{B}\right)$ and subgroups $\left(G_{A 1}, G_{A 2}, G_{B 1}\right.$ and $\left.G_{B 2}\right)$ at different time intervals by 'KruskalWallis Test'. 


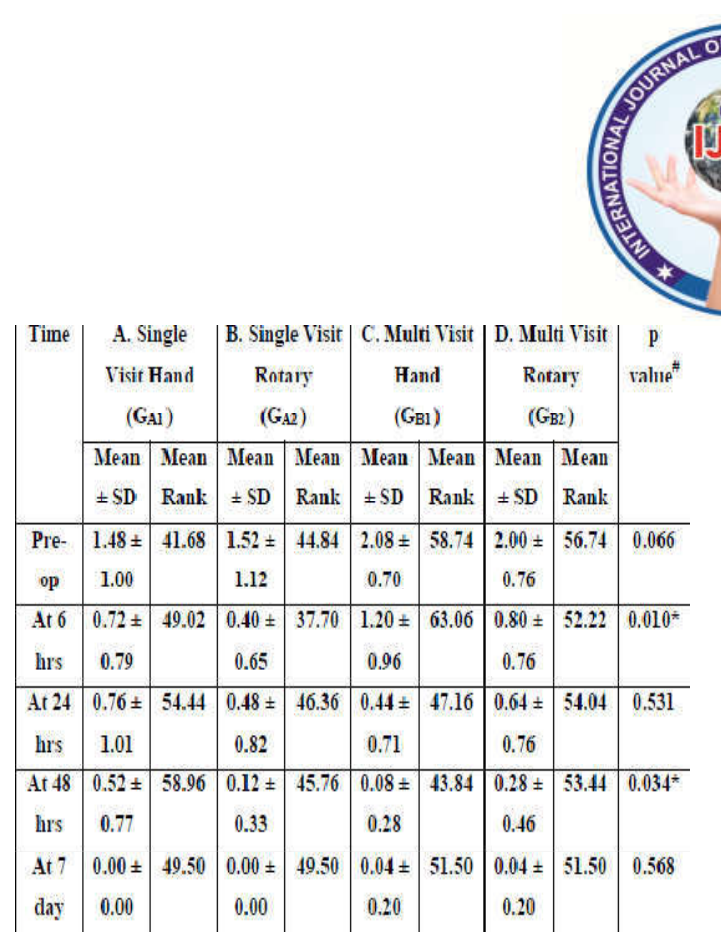

\#Kruskal-Wallis Test; *p<0.05;

Significant

Table 1: shows Comparative evaluation of

incidence of pain in teeth treated in single

visit (subgroup GA1, subgroup GA2) and

teeth treated in two visits (subgroup GB1,

subgroup GB2) at different time intervals.

The results were statistically significant at

6 hour and 48 hour time intervals in

various subgroup combination. $(p<0.05)$.

Table 2: Multiple comparisons of pain using Mann-Whitney Test

\begin{tabular}{|c|c|c|c|c|c|c|}
\hline Time & $\begin{array}{c}\text { Group } \\
\mathrm{G}_{\mathrm{Al}} \text { vs } \\
\mathrm{G}_{\mathrm{A} 2} \\
\text { p value }\end{array}$ & $\begin{array}{c}\text { Group } \\
\mathrm{G}_{\mathrm{Al}} \mathrm{vs} \\
\mathrm{G}_{\mathrm{B1}} \\
\text { p value }\end{array}$ & $\begin{array}{c}\text { Group } \\
\mathrm{G}_{\mathrm{Al}} \text { vs } \\
\mathrm{G}_{\mathrm{B} 2} \\
\text { p value }\end{array}$ & $\begin{array}{c}\text { Group } \\
\mathrm{G}_{\mathrm{A} 2} \text { vs } \\
\mathrm{G}_{\mathrm{B} 1} \\
\text { p ralue }\end{array}$ & $\begin{array}{c}\text { Group } \\
\mathrm{G}_{\mathrm{A} 2} \text { vs } \\
\mathrm{G}_{\mathrm{B} 2} \\
\text { p value }\end{array}$ & $\begin{array}{c}\text { Group } \\
\mathrm{G}_{\mathrm{B1}} \text { vs } \\
\mathrm{G}_{\mathrm{B} 2} \\
\text { p value }\end{array}$ \\
\hline Pre-op & 0.825 & $0.024^{*}$ & $0.044^{*}$ & 0.088 & 0.146 & 0.789 \\
\hline At 6 hrs & 0.127 & 0.074 & n.681 & $0.001 *$ & $0.036^{*}$ & 0.124 \\
\hline At $24 \mathrm{hrs}$ & 0.271 & 0.324 & 0.906 & 0.841 & 0.297 & 0.294 \\
\hline At 48 hrs & $0.035^{*}$ & $0.014^{*}$ & 0.356 & 0.641 & 0.162 & 0.068 \\
\hline At 7 day & 1.000 & 0.317 & 0.317 & 0.317 & 0.317 & 1.000 \\
\hline
\end{tabular}

TABLE 2 shows statistical analysis of change in intensity of post operative pain after various post operative time intervals in subgroup $G_{A 1}$ and $G_{A 2}$, subgroup $G_{A 1}$

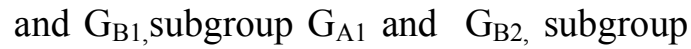
$\mathrm{G}_{\mathrm{A} 2}$ and $\mathrm{G}_{\mathrm{B} 1}$, subgroup $\mathrm{G}_{\mathrm{A} 2}$ and $\mathrm{G}_{\mathrm{B} 2}$, subgroup $G_{B 1}$ and $G_{B 2}$, respectively at different time intervals. The results statically insignificant at all time intervals in various subgroup combinations $(\mathrm{p}>0.05)$ except for subgroup $\mathrm{G}_{\mathrm{A} 2}$ and $\mathrm{G}_{\mathrm{B} 1}$, and subgroup $\mathrm{G}_{\mathrm{A} 2}$ and $\mathrm{G}_{\mathrm{B} 2}$ at 6 hours interval and for subgroup $G_{A 1}$ and $G_{A 2}$, subgroup $\mathrm{G}_{\mathrm{A} 1}$ and $\mathrm{G}_{\mathrm{B} 1}$, at 48 hours interval where results were statically significant $(\mathrm{p}<0.05)$.

\section{DISCUSSION:}

Although a number of studies in the literature show that there is no significant difference between the single visit and multiple visit endodontic treatment as far as incidence and intensity of post-operative pain are concerned, there is a lack of evidence-based data to reinforce this. This can be attributed to the difference in inclusion criteria (vital versus non-vital teeth; single rooted versus multirooted teeth), variability in instrumentation technique, irrigation regimen, sample size, subjective nature of the pain interpretation and evaluation, and pre-operative symptoms of patients etc ${ }^{9}$. Hence, the purpose of this study was to evaluate the postoperative pain after treating teeth with 


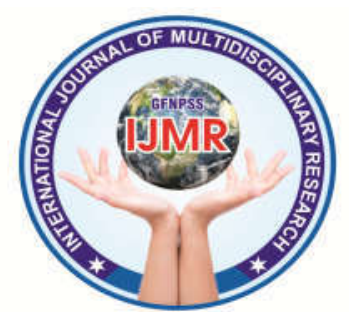

irreversible pulpitis in single and multiple visit using hand protapers and endomotar driven rotary Protaper files. In this study a modified $100 \mathrm{~mm}$ Heft-Parker VAS was used. This is a bounded scale with absolute values at each end and word descriptors of pain levels placed in ascending order along the horizontal axis. Heft and Parker state that the unequal spacing of words on the scale represents an accurate reflection of how patients perceive spacing between the different pain word descriptors. ${ }^{14}$

Time taken to complete the procedure was more for the teeth in which biomechanical preparation was done using hand Protapers than, the teeth in which biomechanical preparation was done using Endomotar and rotary Protapers. This is in agreement with Pasqualini D et al (2008) ${ }^{15}$ who found that the hand protapers required significantly fewer rotation than rotary protapers, whereas the effective working time to fully shape the simulated canals was significantly high with hand protapers. When comparing both groups GA and GB time was more in multiple visits as compared to single visit. The reason may be additional time required for chair preparation, reorientation, isolation and reentry into canal. ${ }^{13}$
In the present study, Table 2 shows statistical evaluation of change in intensity of pain various post operative time intervals in subgroup GA1 and GA2, subgroups GA1 and GB1, subgroups GA1 and GB2, subgroups GA2 and GB1, subgroups GA2 and GB2, subgroups GB1 and GB2 respectively at different time intervals. The results were statistically insignificant at all time intervals in various subgroup combination ( $\mathrm{p}>0.05$ ) except for subgroups GA2 and GB1 and subgroups GA2 and GB2 at 6 hours interval and for subgroup GA1 and subgroup GA2, subgroups GA1 and GB1, at 48 hours interval where results were statistically significant

$(\mathrm{p}<0.05)$.

In single visit group (GA), the teeth in which biomechanical In single visit group (GA), the teeth in which biomechanical preparation was done using Hand Protapers (Sub group GA1) and the teeth in which biomechanical preparation was done using Endomotar and Rotary Protapers (Sub group GA2),(Table 2) there was no significant difference in pain after 6 hours interval and 24 hours but there was significant difference in pain score after 48 hours. 4 teeth had moderate pain in GA1 group which was relieved by taking 


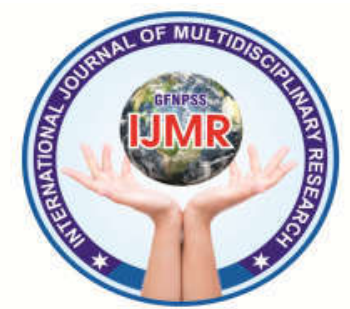

analgesics. Hence, in single visit group intensity as well as incidence of pain after $48 \mathrm{hrs}$ was significantly more in Sub group GA1 than Sub group GA2. The reason for this as per as Marilena Bet al (2012) ${ }^{10}$, Although hand ProTaper instruments represent a revolutionary advance in endodontic treatment, deviation from the principles of mechanical treatment may have a negative effect on prognosis and success rate of treatment ${ }^{10}$.

No pain was observed after a week in both subgroups. More pain was experienced by single visit hand group than single visit Rotary. Also this result is in agreement with Sheetal B Ghivari et al (2011) ${ }^{15}$ The difference in the root canal preparation using hand and rotary Protaper files is because of the time of contact between the file and the root canal wall. The engine-driven Protaper file contacts the apical area for a lesser period of time and also the rotational speed and torque is fixed, whereas, the Hand Protaper file prepares the apical area for an extended period of time and the rotational movement of the file is an "operator controlled variable factor", extruding more amount of debris ${ }^{16}$ While comparing the teeth in which biomechanical preparation was done using Hand Protapers in single visit (Sub group $\mathrm{G}_{\mathrm{A} 1}$ ) and the teeth in which biomechanical preparation was done using Hand Protapers in multiple visits ( Sub group GB1),(Table 2) it was found that there was significant difference in Preoperative pain, here $\mathrm{P}$ value was 0.024 which was $<0.05$. Teeth treated in multiple visit hand protaper group (Sub group GB1) had more preoperative pain than in single visit hand protaper group (Sub group $\mathrm{G}_{\mathrm{A} 1}$ ). After 6 hours interval, there was reduction in pain score and intensity of pre operative pain in both sub groups but it was statistically insignificant. After 48 hours, incidence of pain were more in Sub group GA1 than Subgroup GB1 as 8\% patients in this group had pain in comparison with $36 \%$ in GA1 Subgroup which was significant statistically .

From the above discussion it is clear that overall, As the basic aim of this study was overall comparison of incidence of postoperative pain in single visit and multiple visits root canal therapy. Post operative pain in all cases subsided with use of mild analgesics (ibuprofen) only. In none of the teeth treated in either single visit or multiple visits, more than 3 tablets of analgesic were required in any case. Stronger analgesic was not required. In the AAE survey quoted by Crumpton (1993) ${ }^{11}$ 


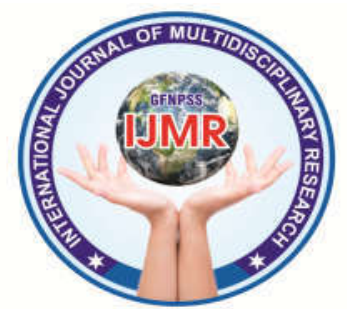

for cases of irreversible pulpitis, $16.76 \%$ of responders prescribed antibiotics. But, in the present study none of the patients developed swelling and so antibiotics were not required in any case. This is in agreement with Haas DA(1997) ${ }^{12}$, Mickel $\operatorname{AK}(2006)^{13}$ who found that endodontic pain is best managed by eliminating the source of infection or inflammation.

\section{CONCLUSION:}

Single visit root canal therapy can be completed in significantly shorter time as compared to Multiple visits root canal therapy.The presence of pre operative pain can significantly influence presence of the post operative pain. As pre operative pain was significantly more in multiple visit root canal therapy, it was same in post obturation pain after 6 hours interval. Most of the pain in both single visit RCT and Multiple visits RCT occurred in first 48 hours after obturation which decrease thereafter. At 6 hours time interval, multiple visit root canal therapy had greater pain as compared to Single visit root canal therapy and difference was statistically significant. In single visit RCT, significantly more post obturation pain was there in single visit hand Protaper group as compared to single visit rotary Protaper group. In multiple visits RCT, no significant difference was found in post obturation pain in multiple visits hand Protapers as compared to multiple visits Rotary Protapers..Hand Protapers subgroups had greater post obturation pain as compared to Rotary Protapers subgroups groups. Pain perception is highly subjective and variable experience modulated by multiple psychosomatic factors. Hence, further studies and researches with larger sample size are required to substantiate the results. Hence more studies required for with different variables.

\section{REFRENCESE}

1. Grossman LI, Oliet S, Del Rio CE. Endodontic practice, $11^{\text {th }}$ ed. Varghese ;1991.

2. Field JW, Gutmann JL, Solomon ES, Rakusin H. A clinical radiographic retrospective assessment of the success rate of single-visit root canal treatment. Int Endod J 2004;37(1):70-82.

3. Sjögren U, Figdor $D$, Persson $S$, Sundqvist G. Influence of infection at the time of root filling on the outcome of endodontic treatment of teeth withapical periodontitis. Int Endod $J$ 1997;30:297-306.

4. Malhotra N, Kundabala M, Acharya S. Contemporary endodontic approac h: 


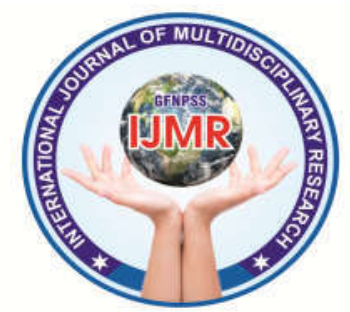

single-visit root canal treatment

endodontics. Clinical Update 2003;25 revisited. ENDO (Lond Engl) 2009;3 (12):23-4.

(3):215-25.

12. Haas DA. Local and systemic therape

5. De-Deus G, Garcia-Filho P,Le Jareiro

R. Influence of the NiTi rotary system on the debridement quality of the root canal space. Oral Surg Oral Med Oral Pathol Oral Radiol Endod. 2009 Oct; 108(4):71-6.

6. Clauder T, Baumann A. Protaper NT System. Dent Clin N Am 2004 ; (48): $87-111$.

7. Ingle JI, Bakland LK. Endodontics. 5th ed. B. C. Decker, Elsevier; 200.

8. Jacob S. Single Visit Endodontics. Fam Prac Dent Handbook 2006;6(4).

9. El Mubarak AH, Abu-bakr NH, Ibrahim YE. Postoperative pain in multiple-visit and single-visit root canal treatment. J Endod 2010;36 (1): 36-9.

10. Marilena Bataiosu, Oana Diaconu ,Iren Moraru, C.Daguci ,Mihaela Ţuculina,Luminiţa Daguci,Lelia Gheo rghiţa. The failures of root canal preparation with hand ProTaper. Current Health Sciences Journal 2012;38,(3):129-31.

11. Crumpton LBJ, McClanahan. Anti biotic resistance and antibiotics in utics for the control of endodontic pain. Alpha Omegan 1997;90(4):73-6.

13. Mickel AK, Wright AP, Chogle S, Jones JJ, DDS, Kantorovich I, Curd F. An analysis of current analgesic preferences for endodontic pain management. J Endod 2006; 32: 114654.

14. Smita Singh, Aniket Garg: Incidence of post-operative pain after single visit and multiple visit root canal treatment. A randomized controlled trial. Journal of Conservative Dentistry-2012; 15(4): 323-27.

15. Pasqualini D, Scotti N, Taman gnone $\mathrm{N}$, Ellena $\mathrm{F}$, Beruttii $\mathrm{E}$. HandOperated And Rotary Protaper Intruments: A Comparision Of Working TimeAnd Number Of Rotations In Simulated Canals. J Enod 2008,34(3);314-7.

16. Sheetal B Ghivari,Girish C Kubasad,Manoj G Chandak, and NR Akarte Apical extrusion of debris and irrigant using hand and rotary systems: A comparative study. J Conserv Dent. 2011 Apr-Jun; 14(2): 187-190. 